\title{
Objeciones textiles: interferencias y activismo textil-digital*
}

\author{
Laura Cortés-Rico** \\ Tania Pérez-Bustos***
}

\section{Resumen}

En este artículo analizamos tres formas en que la materialidad y el hacer textil artesanal interfieren en la manera en que nos relacionamos con el diseño de tecnologías digitales. Primero, la forma en que lo textil sostiene lo digital, revelando redes de trabajo y cuidado que soportan el diseño de tecnologías. Segundo, los modos en que el hacer textil transforma los lenguajes del diseño en ingeniería, permitiendo que las dimensiones afectivas de éste emerjan. Y tercero la forma en que las temporalidades del hacer textil ralentizan valores de eficiencia y productividad propios de la ingeniería.

Palabras clave: Gestos Textiles Materiales, Diálogos de Saberes, Hacer Textil-Digital, Interferencias.

* Recibido el 11 de septiembre de 2019, aceptado el 30 de enero de 2020. El presente artículo es producto derivado del proyecto INV-ING 2981 titulado "Diálogos de saberes en la creación de interfaces físicas artesanales para la interacción con dispositivos móviles" financiado por la Vicerrectoría de Investigaciones de la Universidad Militar Nueva Granada, vigencia 2019, con el apoyo de la Universidad Nacional de Colombia, código Hermes 44835.

** Professora, pesquisadora, Ingeniería en Multimedia, Universidad Militar Nueva Granada, Cajicá, Colombia. laura.cortes@unimilitar.edu.co / ORCID 0000-00017817-6012

*** Professora, pesquisadora, Escuela de Estudios de Género, Universidad Nacional de Colombia, Bogotá, Colómbia. tcperezb@unal.edu.co / ORCID 00000002-2885-2606 
Textile Objections: Interferences and Digital-Textile Activism

\begin{abstract}
In this paper we analyze three ways in which materiality and artisanal textile making interfere how we relate to the design of digital technologies. First, is the way in which textiles sustain the digital, revealing networks of work and care that support the design of technologies. The second involves ways in which textile making transforms the languages of design into engineering, allowing the emergence of affective dimensions of design. And thirdly, the way in which the temporalities of textile making slow the pace of values of efficiency and productivity found in engineering.
\end{abstract}

Keywords: Material Textile Gestures, Knowledge Dialogues,

Digital-Textile Making, Interferences. 


\section{Introducción}

Iniciando el 2016, luego de finalizar uno de los primeros proyectos que realizamos buscando dar cuenta de cómo el quehacer textil artesanal podía posibilitar el encuentro entre diferentes saberes, una periodista científica escribió un artículo reseñando la forma en que habíamos logrado diseñar una interfaz tangible de usuario, en la que los patrones del calado cartagüeño podían representarse computacionalmente usando unas piezas bordadas a mano por las maestras bordadoras de esta región (Fog, 2016). Estas piezas intervenidas artesanalmente, al entrar en contacto con la pantalla táctil de una tableta, activaban una aplicación diseñada por nosotras que les permitía combinar nuevas puntadas para adornar sus prendas. ${ }^{1}$ Era el cierre de un proyecto de muchos aprendizajes. Las bordadoras de Cartago habían participado directamente en la construcción de una tecnología digital, algo que para ellas al inicio era inimaginable. "Esas cosas no son para mi" nos había dicho una de ellas durante el trabajo de campo, refiriéndose al uso de computadoras o teléfonos inteligentes, y luego de 2 años de encuentros cotidianos, habían pasado del miedo y el recelo al entusiasmo. Nosotras, de su parte, habíamos aprendido a bordar dimensionando el conocimiento incorporado que ese quehacer implicaba, habíamos compartido con ellas muchos momentos de intimidad y silencio, pero también de risas provocadas por las exploraciones materiales a las que las habíamos invitado. Juntas habíamos aprendido que no todo lo artesanal podía automatizarse, que había hilos que conducen energía y que ciertas puntadas permiten, mejor que otras, transformar señales físicas en representaciones

\footnotetext{
1 De este trabajo con las bordadoras de Cartago, surgió CalaITU, una interfaz tangible de usuario que consiste en un componente físico (piezas artesanales que se intervienen con hilo conductor) y uno digital (una aplicación móvil instalada en una tableta de pantalla táctil). Cuando las piezas entran en contacto con la pantalla de la tableta que contiene la aplicación, se dibujan representaciones computacionales de las puntadas de calado, que son combinadas para diseñar patrones más complejos (Cortés-Rico, 2015).
} 
computacionales (Tobar Roa, 2015; Pérez Bustos \& Márquez, 2016; Pérez-Bustos, Cortés-Rico, and Márquez-Gutiérrez, 2015).

A pesar del cuidado con el que la periodista había buscado recoger ese proceso de aprendizajes mutuos y comunes, los comentarios sobre la imposibilidad o los peligros de que la tecnología digital se pensara desde el bordado artesanal o viceversa, no se hicieron esperar. "Así es que terminan desapareciéndose tradiciones", dijo alguien en nuestras redes sociales, refiriéndose a cómo el diseño de tecnologías sólo podía expropiar lo artesanal de las manos expertas de quienes lo hacían, automatizándolo y acentuando así las condiciones de precariedad económica de sus hacedoras. La nota de prensa ponía a la gente que la leyó a pensar en que la tecnología intervenía de forma negativa en el quehacer textil hecho a mano. Una asociación que no es gratuita, si tenemos en cuenta que en efecto el trabajo textil es de los que más ha sufrido procesos de automatización (Vidyasagar, 2000). Sin embargo, también es uno de aquellos oficios que ha permanecido haciéndose en el tiempo a pesar de la automatización (Angulo y Martínez 2016). Aunque seguimos tejiendo y bordando a mano, pese a que hay formas más eficientes de producir resultados materiales similares, no eran esos los referentes de los lectores: la tecnología digital y el bordado artesanal no pueden ir juntos sin que lo artesanal desaparezca, nos hacían saber. Y al hacerlo subrayaban una jerarquía construida y particularmente generizada (en el sentido de construida por el género) entre ambas formas de conocer y hacer material. Desde allí, el quehacer textil ocupaba un lugar subordinado, en tanto se concibe como un oficio feminizado, en relación con el diseño de lo digital, entendido como una profesión prestigiosa y masculina (Pérez-Bustos 2016; Plant 1995).

En efecto, el hacer digital interviene el hacer textil y puede hacerlo de formas negativas, pero no solamente. Nuestra intención, como equipo transdisciplinar comprometido políticamente con el reconocimiento de los saberes feminizados con los que trabajamos, siempre ha sido la de buscar intervenciones que sean más fluidas y co-constituyentes, 
reconociendo para ello que el encuentro material entre lo digital y lo textil es, ante todo, de mutuo aprendizaje. En ese sentido, nos ha interesado reiterar que el hacer tecnología digital tiene algo para decirle a quienes hacen labores textiles: que pueden encontrarse con otras manos en la distancia (Pérez-Bustos et al., 2016), que las historias de sus piezas pueden ser conocidas más allá de las piezas mismas (Pérez-Bustos, 2017), que sus puntadas son capaces de amplificar sonidos o crear movimientos (Psarra, 2015; Hannah, 2011), o incluso que ellas pueden transformarse en lenguajes de programación (como es el caso de la primera computadora que surge de un telar (Fernaeus, Jonsson, y Tholander, 2012). Pero también queremos subrayar que el hacer textil nos enseña otro tanto a quienes diseñamos tecnologías: que éste puede sostener el diseño de lo digital, material y humanamente, que puede transformar los lenguajes en que lo digital se expresa trayendo a escena lo afectivo, que permite revelar que los tiempos del diseño digital son tiempos del cuerpo. Nos interesa subrayar que, en ambos casos, estos aprendizajes emergen materialmente en el hacer, se producen en el encuentro que el hacer propicia.

Ahora bien, estos llamados desde afuera a prestar atención a los peligros de la automatización y la expropiación por parte del hacer digital hacia el hacer textil artesanal, como los que provocaron el artículo de prensa del 2016, nos invitan a mantener una reflexividad constante sobre las maneras en que podemos hacernos responsables del encuentro entre estos saberes, para propiciar el reconocimiento y la escucha mutua entre ellos. Es en esa reflexividad que se revelan nuestros aprendizajes como interferencias, producidas por $y$ en el encuentro con el hacer textil. En este sentido, si bien nuestra investigación ha buscado intervenir en esas jerarquías de género creadas entre lo textil y lo digital --en el sentido de que, como veremos en el apartado siguiente, éstas no siempre existieron, sino que son producidas históricamente--, con miras a transformar las maneras en que estos oficios pueden estar juntos, el hacer textil objeto de nuestra investigación, nos interpela de tal forma que interfiere en la 
manera en que lo entendemos e interfiere en la forma en que nos vemos a nosotras mismas en él.

Retomamos aquí el trabajo de Ruth Müller y Martha Kenney (2014) para señalar que las interferencias son asuntos mundanos $e$ involuntarios que tienen la fuerza de perturbar los órdenes establecidos, órdenes de género en este caso, y que son susceptibles de ser percibidos a través de una reflexión situada del propio trabajo investigativo. Siguiendo a estas autoras, mientras que las intervenciones a las que nos referimos anteriormente son intencionales $y$ tienen que ver con procesos de diseño que orientan la manera en que el diálogo de saberes entre lo textil y lo digital se medía, las interferencias, por su parte, se producen en ese encuentro, no se anticipan a él. En este sentido, éstas subrayan la fuerza agencial de la cotidianidad en la que los métodos de investigación están inmersos, para irrumpir y trastocar lo establecido. Más aún, hacen un llamado a prestar atención a aquello que de ellas emerge, "no para producir un inventario objetivo de cómo interferimos [o de lo que interfiere], sino para desarrollar una sensibilidad frente a los efectos que tienen esas interferencias sobre la forma cómo investigamos y podemos llegar a investigar" (Müller y Kenney, 2014:5, nuestra traducción) ${ }^{2}$.

Este artículo versa sobre esas interferencias y de modo particular sobre la forma en que la materialidad y el hacer textil artesanal interfieren en la manera en que pensamos y nos relacionamos con el diseño de tecnologías digitales. En línea con lo ya dicho, entendemos estas interferencias como capaces de trastocar, y en ocasiones deshacer órdenes de género. El trabajo de Karen Barad (2003) es central para dimensionar la naturaleza material de este tipo de interferencias, pues nos invita a comprender que ellas emergen de la relación con las materialidades, y que incluso la agencia de éstas se produce en esa relación, no la precede. En este sentido, el encuentro con lo

\footnotetext{
2 "not to produce an objective account of how we are interfering, but to develop keener sensitivities to the effects of our methods as a way to orient and re-orient our research projects" (Müller y Kenney, 2014:5).
} 
textil para pensar el diseño de tecnologías es el escenario en el que las materialidades textiles van a decirnos algo sobre las prácticas de quienes diseñan tecnologías digitales, sus formas de hacer, la disposición de sus cuerpos y la relación de estos con otros cuerpos. Ello nos permite dimensionar otras formas de imaginar la relación que nuestras prácticas de investigación establecen con el mundo y en ese sentido nos permiten construir mundo también (Haraway, 2013).

Nos interesa argumentar que esta sensibilidad, producto de la reflexión sobre lo que hacemos, es una forma de activismo feminista, porque nos invita a comprometernos con nuestras prácticas de investigación, a hacernos responsables no sólo de lo que ellas son, sino de lo que podrían llegar a ser (Galloway, 2013). Para dar cuenta de estas interferencias recogemos etnográficamente momentos de nuestro trabajo investigativo, en los que al encontrarnos material y transdisciplinariamente en el hacer textil, van emergiendo formas en que éste objeta la manera en que concebimos las tecnologías digitales, así como los valores, lugares y prácticas de quienes las diseñan desde la ingeniería. Es este foco en el hacer textil material el que nos lleva a llamar a estas interferencias objeciones textiles.

En este artículo realizamos este análisis reflexivo, en una escala personal, en dos movimientos. En el siguiente apartado, la materialización textil de la información, presentamos ejemplos de otras investigaciones en las que lo textil y lo digital se hacen de forma mutua. Estos ejemplos sirven también para mostrar que la dicotomía entre estos saberes-haceres y la forma en que ella está constituida por el género, es producto de una construcción, que no siempre ha operado funcionalmente. En el ejercicio de presentar estos antecedentes nos interesa resaltar la manera en que ellos han inspirado nuestro propio trabajo investigativo. En la sección objeciones textiles, por su parte, nos concentramos en tres interferencias que presentamos desde viñetas etnográficas: (1) la forma en que lo textil sostiene lo digital, consiguiendo revelar las redes de trabajo y cuidado que soportan el diseño de tecnologías; (2) los modos materiales en que el hacer textil transforma los 
lenguajes del diseño en ingeniería y permite que las dimensiones afectivas de éste emerjan; y (3) la forma en que las temporalidades del hacer y el deshacer textil ralentizan valores de eficiencia y productividad propios de la ingeniería dando pie para reconocer que el diseño puede orientarse por valores más encarnados.

\section{La materialización textil de la información}

Desde el vestuario hasta la computación, pasando por la revolución industrial, la relación que existe entre lo textil como artesanal y lo textil como tecnológico ha sido controversial. En el centro de esta disputa el quehacer textil se ubica simultáneamente como asociado a lo tradicional, lo ancestral y lo hecho a mano (Arnold y Espejo, 2013), así como centro de interés y punto de partida para el desarrollo industrial y la automatización (Styles, 2016; International Labour Organization, 2000). Allí, lo textil representa lo particular de una cultura, sus personas, sus ecologías (Jones, 2001; Arnold y Espejo, 2013), un saber que es necesario preservar (Ebert et al. 2014); pero a la vez, la industrialización y la globalización traen consigo procesos en serie de creación masiva de piezas textiles (Nordås, 2004), en los que las personas que participan de su creación son completamente invisibilizadas por el mercado (Dedeoğlu, 2010).

Estas controversias en ocasiones no permiten entender que lo textil artesanal es en sí mismo una tecnología, pero incluso que la tecnología tiene una dimensión artesanal que también le constituye. En este apartado recogemos diversos ejemplos que buscan problematizar estas tensiones y dar cuenta de cómo lo "hecho a mano" y lo "hecho a máquina" están profundamente entretejidos.

La computación, en sentido estricto, se basa en el estudio de la representación y el procesamiento de la información (Science Museum, 2018). En sus inicios, se relacionaba esencialmente con cálculos matemáticos simples de conteo y operaciones aritméticas de adición y diferencia, pero también con el ordenamiento de datos; por ejemplo, se contabilizaban transacciones económicas de 
intercambio de productos, registrando cantidades, fechas y personas involucradas en el intercambio (Turing, 2018). Este tipo de trabajo ha ido escalándose para llegar a la construcción de algoritmos complejos utilizados en diversos campos como la inteligencia artificial, el aprendizaje de máquina, la analítica de datos, el procesamiento de imágenes, entre otros muchos. Por ejemplo, actualmente no solo se registran transacciones económicas, sino que se analizan computacionalmente comportamientos estadísticos de los datos para anticipar las tendencias del mercado. Estos algoritmos complejos han llegado a una escala de cómputo en la que se reduce la intervención humana, de forma que se realizan análisis prescriptivos de la información para tomar decisiones con base en el comportamiento de grandes cantidades de datos. Ahora bien, independientemente de la escala, todos los procesos computacionales se basan en operaciones aritméticas y lógicas simples ${ }^{3}$. Nos interesa subrayar aquí que las formas en las que se representa la información y en las que se llevan a cabo esas operaciones simples, han estado históricamente ligadas con el hacer textil, a pesar de que ese vínculo no sea de conocimiento común. A continuación, presentamos algunos referentes no exhaustivos en los que se evidencian estas convergencias, reflejando cómo la información ${ }^{4}$ se materializa a través de técnicas, herramientas y elementos textiles. Con esto buscamos contribuir localmente a la deconstrucción de las dicotomías que se han establecido entre estos saberes-haceres y los sesgos de género que las sustentan.

Uno de los primeros casos de los que se tiene información histórica es el Quipu, un artefacto Inca, que data del 3800 A.C (Fotografía 1). El Quipu consistía en anudar hilos de diferentes

\footnotetext{
3 La arquitectura de los computadores modernos se basa en un conjunto reducido de instrucciones (RISC) que al ser combinadas de forma ordenada, permiten la creación de algoritmos para la solución de problemas computacionales complejos (Patt 2001).

4 En este artículo comprendemos el concepto de información como el de conjuntos de datos que, en un contexto particular, adquieren un significado.
} 
colores (extraídos de y tinturados con animales y plantas andinas) sobre un trozo de madera para representar información de distinto tipo, como cantidades en sistemas numéricos posicionales ${ }^{5}$, registros de transacciones económicas como trueques de alimentos realizados entre miembros de la comunidad o posesión de tierras, $e$ incluso historias de la cotidianidad Inca, donde los nudos, colores y posiciones se utilizaban para representar sucesos de la historia (Thiruvathukal, 2011).

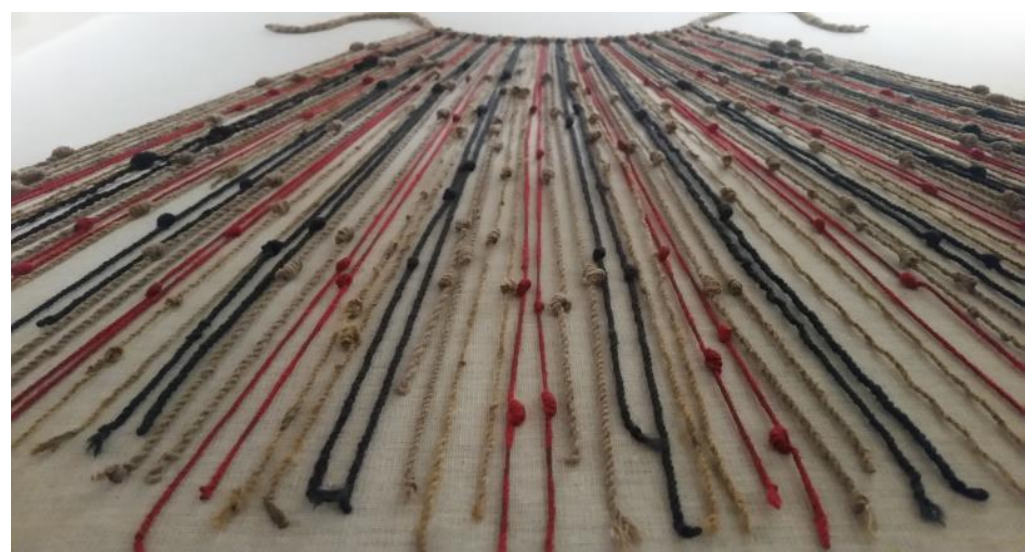

Fotografía 1. Imagen de un tejido Quipu, en la que se observa que la representación material de la información se realiza a través de colores y nudos. Fuente: Museo Machu Pichu, Casa Concha.

En términos computacionales, el Quipu significó disponer un almacenamiento material de la información, donde cada nudo, aunque construido con la misma técnica, tenía un significado diferente de acuerdo con su posición y color, equivalente a lo que

${ }^{5}$ Los sistemas numéricos posicionales son considerados una técnica compleja de conteo, ya que con una cantidad limitada de símbolos (por ejemplo, los números del 0 al 9 en el sistema decimal), es posible representar infinidad de cantidades. Esto debido a que el "peso" cuantitativo del símbolo (número) depende de su posición. Por ejemplo, el número 444 aunque tiene el mismo símbolo (4) tres veces, representa valores diferentes (400, el 4 de más a la izquierda, 40 el 4 central y 4 el de más a la derecha). 
en tecnología digital moderna sería un dígito binario, un bit ${ }^{6}$. En este sentido, cada nudo como entidad independiente, es solo el entrecruzamiento de una cuerda consigo misma; pero al ser combinado en un textil con miles de nudos -se han encontrado Quipus con más de 2000 cuerdas, cada una con varios nudos (Thiruvathukal 2011)-, se logra representar información compleja que a los Incas les permitía anticipar fenómenos naturales como sequías o desastres (Tierra Libre, 2008).

Otro clásico ejemplo histórico de materialización textil de lo computacional es el telar de Jacquard, que inspiró a Babbage a inicios del siglo XIX a diseñar la que es considerada la primera computadora de la historia. El telar de Jacquard se encontraba compuesto por una serie de tarjetas perforadas cuya perforación (o no) permitía el paso (o no) de la aguja. Cuando la aguja pasaba, la urdimbre se tejía. La variación de colores en los hilos, así como la utilización de varias tarjetas con perforaciones distintas, permitía la creación textil de patrones complejos (Fotografía 3). Esta forma de tejido fue la primera manera de programar: modificar comportamientos a partir de información almacenada (en este caso, las perforaciones en las tarjetas). Este telar era conceptualmente muy similar a las computadoras modernas: los pasos para tejer el textil se podían representar como datos a través de tarjetas perforadas que se podían modificar pues la máquina era programable- (Fotografía 2), las tarjetas se guardaban en un almacén -equivalente a una memoria-, existía un lugar específico para realizar el tejido -como la unidad de procesamiento-, y la interacción con las personas era a través de unidades de entrada y de salida, sin requerir intervención humana directa durante el proceso directamente (Turing, 2018; Patt, 2001).

\footnotetext{
6 Unidad mínima de información empleada en los procesos informáticos. El bit es una entidad independiente de 1 y 01 que al combinarse soportan algoritmos complejos o procesos simples de encendido o apagado.
} 

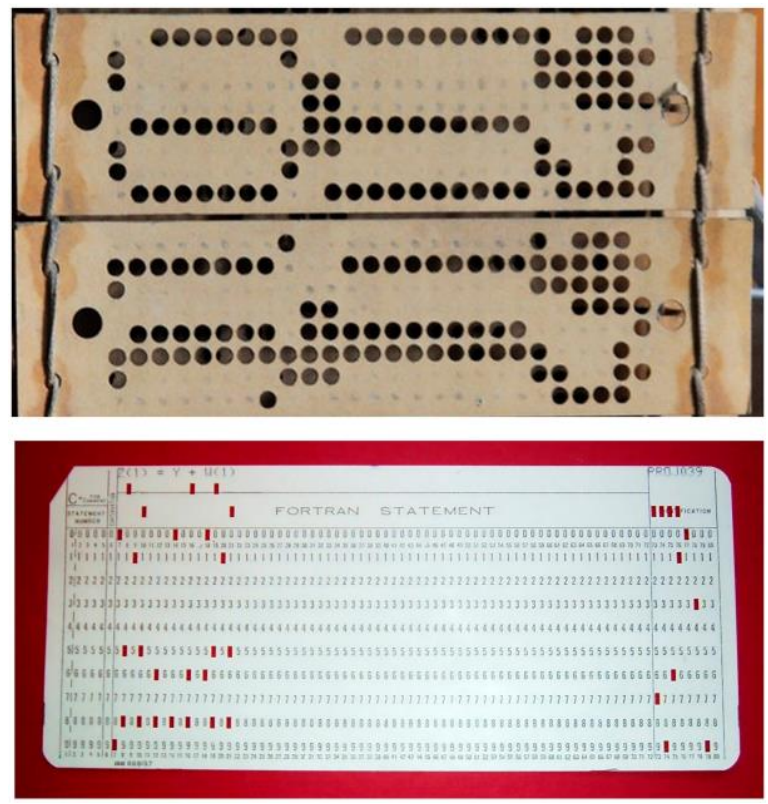

Fotografía 2. Superior, tarjeta perforada usada en el telar de Jacquard para computar información en el telar, sobre patrones de tejido. Fuente: Museum of Science Industry. Inferior, tarjeta perforada Fortran utilizada para computar información digital. Fuente: Wikipedia

La propuesta de Babbage, que se inspiró en el telar de Jacquard, consistía en hacer uso equivalente de las tarjetas perforadas para representar secuencias de instrucciones, permitiendo variar la forma en que se computaba la información de manera equivalente a como se podían programar los patrones de tejido en el telar. Ada Lovelace, quien trabajaba con Charles Babbage, fue quien anticipó el enorme poder computacional de una máquina como estas, con la que se pueden representar simbólicamente infinidad de cosas (notas musicales, partes de una imagen, fechas, textos), más allá de solo cantidades numéricas (Fotografía 3). Ella es considerada la primera persona en escribir un programa de computador, al diseñar las perforaciones de una serie de tarjetas para computar la secuencia numérica de Bernoulli (Turing, 2018). De acuerdo con Romero Sánchez (2014) su trabajo 
fue inicialmente ocultado tras el de Babbage, aun cuando proponía mejoras para el buen funcionamiento de la máquina. Lo que deja ver la forma en que el género tuvo un papel central en la invisibilización de las relaciones entre el hacer textil que inspiró a esta matemática y los orígenes de la computación moderna. A pesar de esto, lo textil como lenguaje de programación es el aporte más importante para la computación hoy en día y se basa en la comprensión del tejido como el entrecruce de urdimbre y trama.

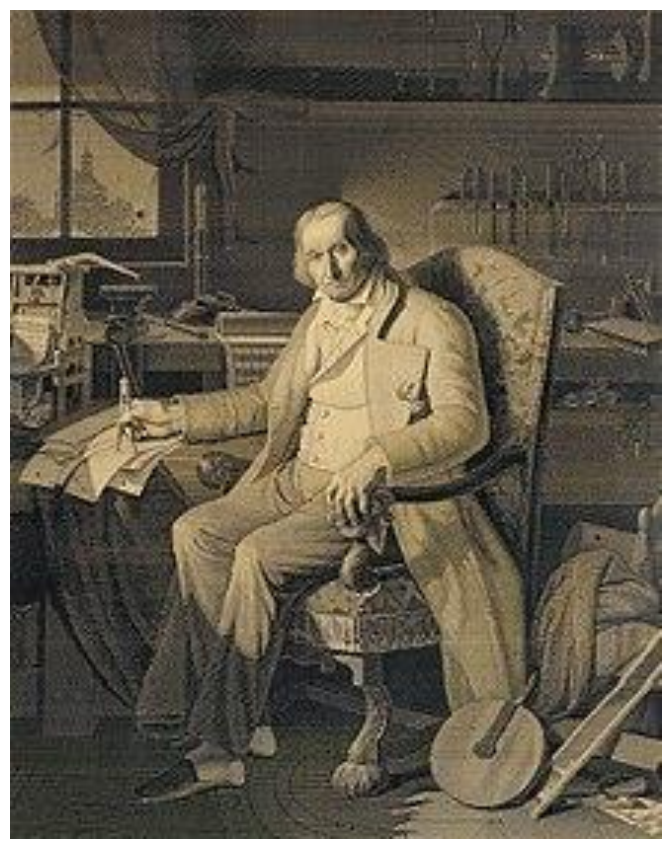

Fotografía 3. Esta imagen corresponde a un retrato de Jacquard tejido con un telar. Realizarlo, requirió más de 24000 tarjetas perforadas (Turing 2018). Fuente: Wikimedia.

Las memorias de núcleo magnético corresponden al tercer ejemplo que nos interesa traer aquí para dar cuenta de los entretejidos entre quehacer textil y tecnología digital. Estas memorias guardaron la información digital usada en la misión 
Apolo de la NASA, de manera que cada porción de información (bit) fue manualmente tejida por mujeres (Rosner et al. 2018). La información estaba asociada con sistemas de guía, independientes de los sistemas control en la Tierra, que permitieran autonomía de los sistemas de cómputo a bordo.

Las tarjetas perforadas, que se habían utilizado hasta ese momento como sistema de almacenamiento de la información, eran muy grandes y pesadas, haciendo inviable este sistema para viajar con la tripulación. En este sentido, la maleabilidad y suavidad de lo textil apareció de nuevo como una solución para las tecnologías computacionales, y se propusieron estas memorias (Fotografía 4). En ellas, el cable era tejido manualmente alrededor o a través de un pequeño núcleo magnético; que, según la técnica, materializaba la representación de un 1 o un 0 . La labor de tejido debía ser cuidadosa, de un lado porque requería hacerse manualmente por el tamaño de los núcleos y de otro, porque era necesario evitar errores. El cuidado que las mujeres tejedoras pusieron en la fabricación de estas memorias logró reducir los errores a 1 en 3 mil millones (Tomayko, 2000). Sin embargo, la confiabilidad en la construcción manual era baja, y las tejedoras de software tenían inspección de su trabajo todo el tiempo (Fildes, 2009). Aunque central y determinante en la misión Apolo, esta labor textil fue invisibilizada, e incluso despreciada; las mujeres tejedoras eran llamadas "LOL - Little Old Ladies-" por los ingenieros de la misión, siendo en general desconocido su trabajo por la historia de la primera misión tripulada a la luna (Rosner et al. 2018). 


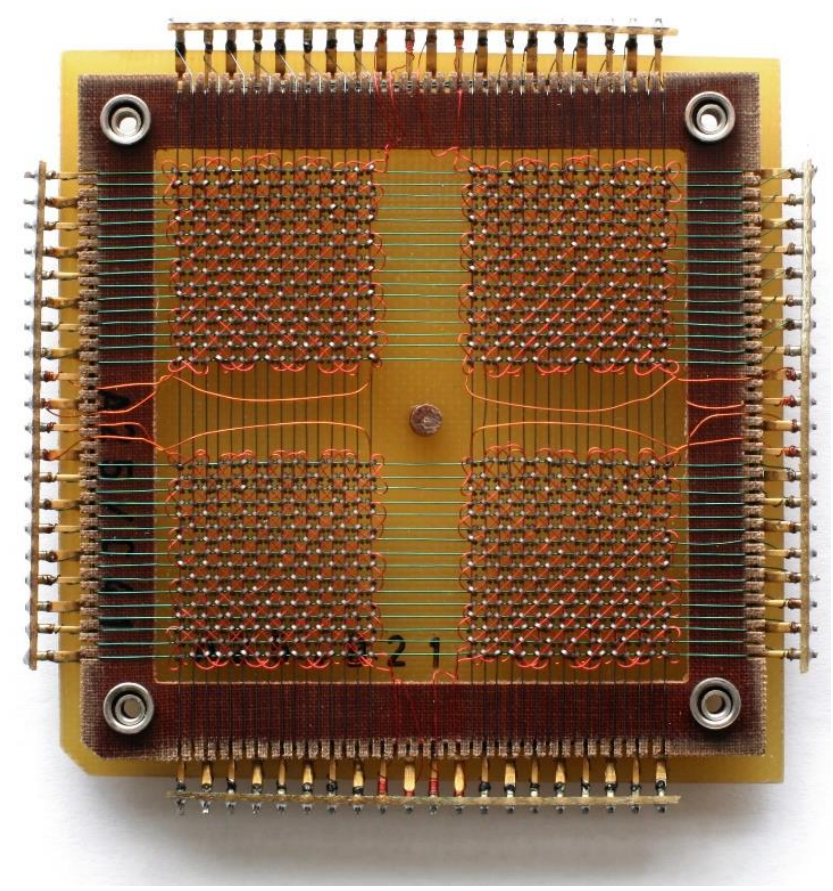

Fotografía 4.Imagen de una memoria de núcleo magnético, con el cable entretejiendo la materialización de la información. Fuente: Museo de Información Histórica.

Las intervenciones entre lo textil y lo computacional no sólo aparecen en el pasado. En la actualidad, son diversos los referentes en los que este hacer artesanal da forma a lo computacional. Este es el caso de la computadora bordada por el equipo de la Universidad de Artes Aplicadas de Viena, liderado Irene Posch y Ebru Kurbak. Este artefacto consiste en una instalación que hace uso de materiales textiles, como hilo de oro o apliques magnéticos, que se bordan en patrones específicos sobre un textil, para realizar procesos de cómputo de información sin utilizar componentes de hardware de estado sólido (Posch, 2016). La información materializada a través de elementos textiles en este caso, se utilizan para construir pequeñas compuertas artesanales. 
Una compuerta es un elemento computacional que permite realizar operaciones lógicas (a nivel de "1s" y "0s"). Las compuertas que se usan en la electrónica actual se realizan con elementos de estado sólido como transistores, y son el componente fundamental de los computadores. Lograr una compuerta textil implica que es posible construir textilmente y por completo un computador de propósito general. Más allá del reto técnico que supone este artefacto, la computadora bordada es una declaración política material sobre cómo lo textil artesanal es computacional en sí mismo, no un referente del pasado, una posibilidad funcional y real del ahora.

\section{Objeciones textiles}

Las diferentes materializaciones textiles de la información, como principio computacional, a las que hemos referido en el apartado anterior, sustentan las exploraciones ingenieriles y etnográficas que han caracterizado nuestra investigación transdisciplinar de los últimos años. En este apartado damos cuenta de cómo esos diálogos con y desde lo textil-digital en su dimensión material han contribuido a destejer cotidianamente la controversia que ubica a estos quehaceres, textiles y digitales, en orillas opuestas y generizadas; a pesar de los múltiples ejemplos históricos que señalan sus interdependencias. Para ello recogemos, etnográficamente, tres momentos de nuestro trabajo investigativo desde los que van emergiendo formas en que el hacer textil objeta la manera en que concebimos las tecnologías digitales, así como los valores, lugares y prácticas de quienes las diseñan desde la ingeniería.

\section{Sostener}

En nuestras exploraciones, el lugar de las telas siempre ha ocupado un papel fundamental, pero en ocasiones invisible, frente a la forma en que buscamos que las materialidades textiles y digitales se encuentren. Las telas soportan el trabajo digital que 
hacemos con hilos conductores de electricidad, permitiendo que éste pueda bordarse y convertirse en un circuito o sosteniendo puntadas de bordado que son luego reconocidas por pantallas táctiles (Fotografía 5). ¿Qué significa esta tarea textil de sostener lo digital? Por un lado, significa que sus características materiales orientan de formas particulares lo que es posible hacer con ellas. Al ser flexibles y livianas, las telas pueden ser atravesadas por agujas y sostener componentes digitales pequeños, como hilos conductores o microprocesadores que se prenden a ésta con puntadas básicas de costura, ello, también permite que estos componentes, con su sostén, puedan luego agregarse a otras telas y permitir que ellas midan temperaturas corporales o produzcan iluminación. Cuando comenzamos a trabajar con bordadoras, esta posibilidad de usar la tela para sostener lo digital les abría la imaginación con facilidad. Dejaban de pensar en lo digital como algo pesado o externo a sus cuerpos o cotidianidades, para imaginar que estaba en la ropa de bebé que ellas elaboraban y que podía anunciar si una pequeña tenía fiebre o se imaginaban que sus manteles de navidad podían adornarse con bordados que iluminaran. 


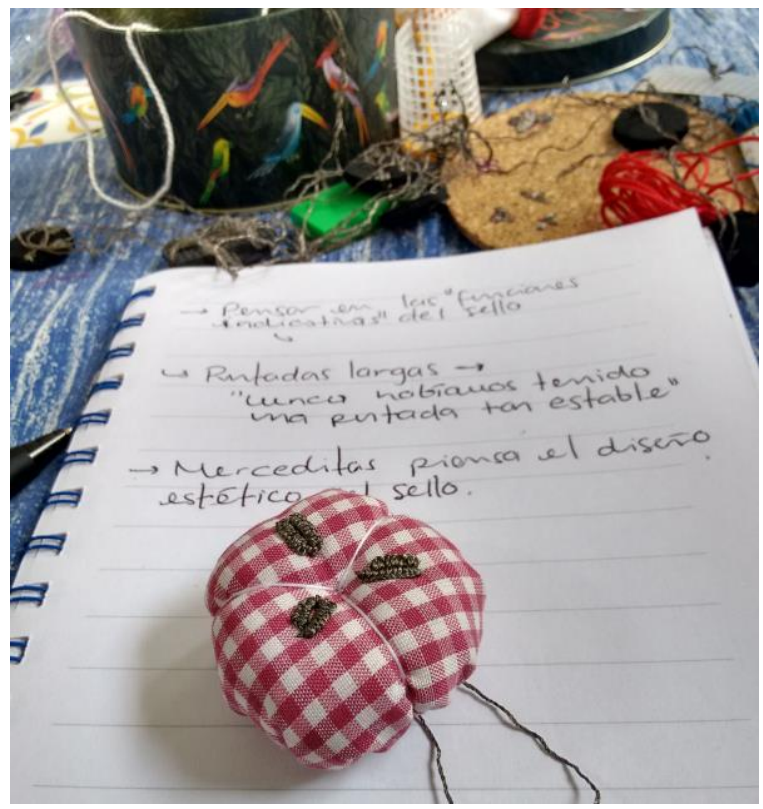

Fotografía 5. Puntadas de bordado sobre cojín de tela hecho por una bordadora como ejercicio para interactuar con una pantalla táctil. Atrás se ven otros retazos de hilos conductores y no y su uso sobre otras superficies. Fuente: Registro visual de nuestro trabajo de campo.

Este interés por parte de las bordadoras sobre cómo las telas podían sostener lo digital, también las llevaba a querer explorar esas posibilidades. Lo que va a subrayar una segunda forma de entender lo que significa que lo textil sostenga lo digital. Nos referimos aquí al trabajo humano que hace posible que una materialidad digital se incorpore en una textil o viceversa. Así, no se trataba de pensar lo digital desde lo que ellas hacían, sino de hacerlo con ellas y en ese hacer pensar con ellas lo que lo digital podía llegar a ser (Lindström y Ståhl, 2016). Bordar con ellas las telas usando hilos conductores nos llevaba a aprender que no todas las puntadas conducían electricidad de igual forma, pero también nos permitía entender que la flexibilidad de la tela podía 
en ocasiones desfigurar estas puntadas y que ellas se reconocieran con menor facilidad por las pantallas táctiles. El trabajo de bordar hacía posible, sostenía, literalmente, lo digital. Las manos de las bordadoras cosían cuidadosamente cada componente, del mismo modo en que las mujeres de la misión Apolo tejieron con sus manos las memorias de núcleo que hicieron posible el primer viaje a la Luna (Rosner et al., 2018). En ese hacer había conocimientos incorporados que permanentemente cuestionaban nuestros supuestos sobre cómo lo textil podía sostener lo digital, esos cuestionamientos, aunque no siempre conscientes, llamaban nuestra atención sobre el trabajo cuidadoso de esas mujeres y la forma en que éste también sostenía el nuestro (Suchman, 2009). Y decimos que no siempre conscientes porque a pesar de que era un aprendizaje que se producía en el hacer juntas sobre la mesa del comedor de sus casas, compartiendo allí formas concretas de resolver los requerimientos que habíamos llegado a definir en conjunto, no siempre terminaban de saber para qué estábamos haciendo todo esto y les costaba mucho trabajo dimensionarse como parte del proceso. Esto era algo que hacían otras personas, no ellas y esa era una forma en la que ellas mismas volvían invisible su papel de sostenernos.

En la formación y práctica de la ingeniería, y en particular de la ingeniería de sistemas, software y computación, este diálogo material encarnado cotidianamente no es usual; en su lugar, se priorizan encuentros esporádicos con las personas que se encuentran en situaciones denominadas "problemáticas" para intentar modelar dichas situaciones y proponer soluciones técnicas. Estos encuentros son mediados por esquemas y modelos que tienen como objetivo anticipar cómo va a ser el artefacto tecnológico que modifica esa situación (Dieter y Schmidt, 2012). Es desde allí que se definen una serie de requerimientos, a manera de lista de chequeo, que luego se usan para evaluar el funcionamiento del artefacto resultante. En este proceso, las personas con las que se interactúa desde la ingeniería tienen una participación puntual que resulta invisibilizada, atribuyendo la 
creación tecnológica solo al equipo de ingeniería, o a quien lidera ese equipo (Rosner, 2018).

La relación de creación tecnológica que tuvimos con las bordadoras fue muy diferente: el trabajo de bordar telas que sostenía materialmente hilos conductores y otros componentes electrónicos, permitió develar esas infraestructuras humanas y más que humanas que sostienen otros procesos de ingeniería, incluso si no son siempre visibles. En ese sentido, el articular el proceso de diseño en su cotidianidad, haciéndolas partícipes de nuestras exploraciones, objetó nuestra concepción del diseño de artefactos tecnológicos en general como algo que sucede desde ninguna parte (Suchman, 2002) y nos llevó a pensar que incluso allí los procesos de ingeniería tienen cuerpo, que ese cuerpo es siempre múltiple y que no son sólo ingenieras e ingenieros quienes los encarnan.

En el trabajo con las bordadoras cartagüeñas había un llamado permanente a que nuestro cuerpo hiciera con ellas el bordado de las interfaces que estábamos intentando pensar. Allí, no era posible proyectar requerimientos, teníamos que irlos descubriendo en el contacto corporal y cotidiano con esas mujeres. Esa convivencia en espacios domésticos, desestabiliza una idea de ingeniería que se centra en crear artefactos funcionales para personas abstractas y subraya que ese proceso emerge de un trabajo encarnado, un trabajo de cuerpos en relación y contacto permanente; cuerpos atravesados por el género.

\section{Transformar}

Luego de terminar el proyecto con Cartago, al que referimos en la introducción y en el apartado anterior, tuvimos la oportunidad de continuar con nuestro trabajo exploratorio a través de una investigación conjunta con el profesor Dominique Vinck de la Universidad de Lausane. En esta pesquisa buscábamos propiciar prácticas pedagógicas interculturales en las que el trabajo artesanal y tecnológico pudieran bordarse mutuamente. Este 
proyecto fue un fondo semilla para seguir con nuestra investigación y nos permitió amplificar lo que estábamos haciendo para poder seguir pensando desde otros haceres textiles lo que la tecnología digital era y podía llegar a ser. Para esto creamos un espacio de encuentro quincenal que llamamos el costurero experimental, en el que nos reunimos con otras mujeres interesadas en explorar materiales y hacer lo textil, pero sobre todo en juntar cosas que usualmente no juntamos. Todas éramos académicas, así que reunirnos a coser y experimentar era algo que nos sacaba de la rutina del trabajo frente al computador, pero así mismo era un espacio al que era difícil encontrarle tiempo. Juntábamos falta de tiempo con un hacer que implicaba cuidado y tomaba tiempo y juntábamos materialidades textiles y digitales en ese hacer cuidadoso.

Una de las sesiones más significativas de este costurero fue cuando propusimos hacer la bobina de un parlante con bordado. Decimos significativo pues este hacer quedó en nuestra memoria y luego hizo parte de otros proyectos en los que le utilizamos para propiciar la reflexión sobre lo que conlleva escuchar y cómo el hacer textil-digital puede construir cuerpos que puedan escuchar con cuidado mensajes que lo requieren (como es el caso de aquellos enviados por comunidades en medio del conflicto). El ejercicio era sencillo: debíamos hacer una espiral con espiras muy cercanas, bordada con hilo conductor de modo que su principio y final estuvieran sueltos, y que ninguna espira se tocara con otra; las partes de hilo sueltas debían conectarse a una fuente de sonido amplificada (un celular, por ejemplo). Al pasar por la espiral y acercar un imán, la señal digital del celular se transformaba en un movimiento de la tela que producía, a su vez, el sonido.

El efecto de este hacer fue conmovedor. Nos tomó horas bordar la espiral, buscando que el hilo no se tocara en ningún punto consigo mismo, y que ello redujera la longitud efectiva de la bobina. En varias oportunidades tuvimos que deshacerla, pues cuando ensamblamos la espiral con los otros componentes del parlante, éste no vibraba lo suficiente para producir sonido. El bordar nos puso en un silencio que nos permitía escuchar nuestro 
hacer, a veces conversábamos mientras hacíamos la espiral sobre cómo nos sentíamos, otras veces nos reíamos cuando a alguna le salía algo mal y nos ayudábamos a resolver el problema. Eran todos contactos íntimos y cercanos, pues la única manera de entender lo que pasaba era a través del contacto mutuo desde lo textil (Pérez-Bustos; Tobar Roa; Márquez Gutiérrez, 2016). Cuando el primer bordado estuvo listo y pudimos escuchar en él el mensaje, la emoción fue colectiva. Al ensamblar los componentes, la tela comenzó a vibrar muy sutilmente y a calentarse, el sonido ya no era una señal digital era movimiento y calor, un lenguaje corporal y sutil que permitía que pudiéramos escuchar como un susurro el sonido que estaba grabado en el celular.

Esta intimidad producida en el hacer textil objetaba de formas muy directas el saber-hacer de la ingeniería. Como disciplina, ésta se encuentra estrechamente relacionada con la experimentación y la aplicación de conocimientos en soluciones técnicas. De acuerdo con el instituto ABET, encargado de acreditar internacionalmente los programas de formación en ingeniería, las habilidades que se aprenden en ésta incluyen, entre otras, "la capacidad de realizar pruebas y mediciones estándar y de realizar, analizar e interpretar experimentos" (2018). En procesos de aprendizaje, estos experimentos suelen estar guiados por la teoría, y buscan verificar en el laboratorio ciertos postulados teóricos, que se consideran verdades. Así, en la investigación en ingeniería el diseño de experimentos consiste en organizar de manera sistemática una serie de pruebas que lleven a afirmaciones estadísticamente significativas. La experimentación busca evaluar, predecir, entender, controlar y mejorar (hacer más eficiente) un proceso o producto (Basili, Selby y Hutchens, 1986). El cuerpo de quienes experimentan desaparece.

El trabajo en el costurero experimental fue distinto. Allí nos reuníamos a especular en torno al hacer, a encontrarnos alrededor de materialidades textiles y digitales con un propósito más cualitativo que cuantitativo, diríamos incluso casi que afectivo. Un hacer anclado en el proceso mismo de experimentación en colectivo, donde la obtención de resultados tenía un lugar 
marginal. Así, la experimentación trascendió los límites temporales y espaciales del encuentro, y nos permitió sensibilizarnos acerca de las sinergias entre lo textil y lo digital y con ello entre nosotras mismas. Como lo mencionamos anteriormente, lo que allí realizamos quedó en nuestra memoria corporal y fue retomado en un proyecto posterior, en el que bordamos parlantes con mujeres que conforman colectivos de memoria en torno a la costura, en contextos de postconflicto, y quienes se encuentran con otras mujeres a través de la escucha, usando parlantes que ellas mismas bordan? ${ }^{7}$.

En el costurero experimental construimos parlantes que tenían un volumen muy bajo. Desde la ingeniería, este parecería un problema de experimentación: no se evaluaron las variables suficientes para lograr un buen volumen con los parlantes, los materiales textiles causaban pérdidas energéticas que no permitían la transformación de la señal eléctrica en una vibración lo suficientemente audible. Técnicamente, un buen parlante es aquel que genera pocas pérdidas en el proceso de transformación de la señal eléctrica, en la señal acústica, y aquél que permite una alta relación señal a ruido. Un proceso técnicamente riguroso de construcción de parlantes, además, implica la estandarización en su diseño. Así, se busca evitar la aleatoriedad en su comportamiento, manteniendo igual el número de vueltas de las bobinas -espirales-, el calibre del alambre utilizado, la distancia entre las espirales, y la membrana -tela en el caso del parlante artesanal. Sin embargo, las materialidades textiles tenían un comportamiento poco controlable desde lo experimental: las espiras no son perfectas, bordar más espiras y más juntas sin que se toquen toma mucho tiempo, cuando la tela suena, sus vibraciones y temperatura son otras formas en las que se transforma la energía. Estas cualidades textiles, en vez de ser un problema experimental, constituyeron el gesto clave con las

\footnotetext{
7 "Remendar lo nuevo: practicando reconciliaciones a través del quehacer textil y la memoria digital en la transición al postconflicto de la Colombia rural", más información: http://artesanaltecnologica.org/remendar_lo nuevo/
} 
mujeres de los colectivos del proyecto Remendar lo nuevo (Fotografía 6); la escucha es un proceso cuidadoso, difícil, que requiere disposición y tiempo.

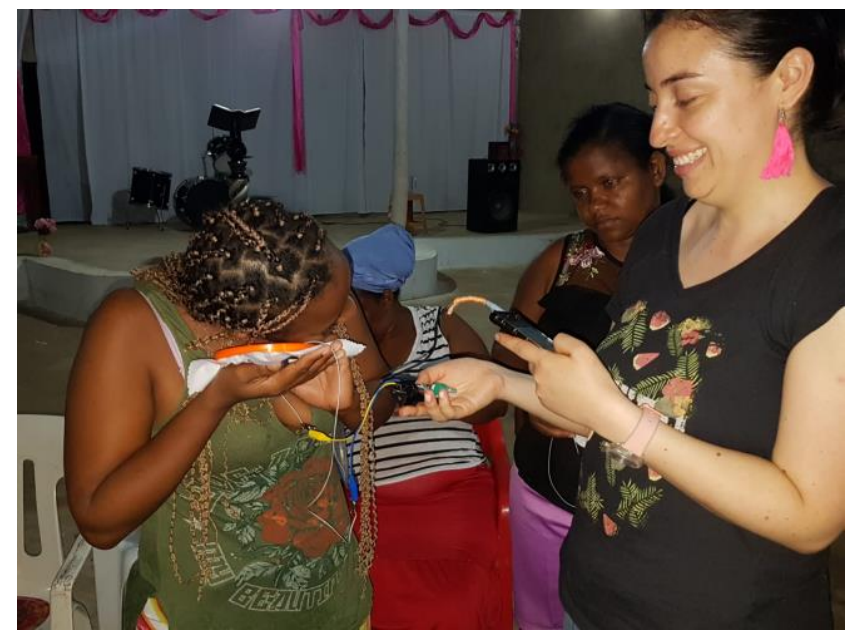

Fotografía 6. Izquierda, gesto de una de las mujeres del colectivo de Mampuján, del proyecto Remendar lo nuevo, al escuchar un mensaje a través de su parlante bordado. Derecha, Alexandra, usando su celular como fuente de audio para reproducir el mensaje que suena en el parlante. Fuente: Registro visual de nuestro trabajo de campo.

\section{Ralentizar}

El hacer textil artesanal toma tiempo, demanda una cierta presencia y disposición corporal. Es repetitivo y configurativo, lleno de pequeños movimientos que se van vinculando uno sobre otro y que en ese anudar cuidadoso van creando superficies o interviniéndolas (Ingold, 2007). Es el cuerpo el que anuda y repite con cuidado, ello mantiene ocupado al pensamiento en una tarea que requiere concentración, pero que, a base de repetición, parece trascender el plano más inmediato de lo tangible y la acción física, por eso se siente como una meditación y convoca tanto al silencio. A veces la repetición conduce a errores y ello implica que es preciso desbaratar y volver a hacer. Así, el hacer 
textil artesanal trae consigo un permanecer en ese hacer-deshacervolver-a-hacer, hasta que el trabajo se termina, o hasta que se deja abandonado por ahí, pues el tiempo no alcanzó para darle feliz término. Cualquiera sea el caso, ese tiempo creativo permanece guardado en el cuerpo y se expresa en futuras creaciones o aprendizajes.

Como contábamos en el apartado anterior, hacer las bobinas bordadas nos tomó horas, entre otras razones porque nos quedaba mal la espiral y teníamos que volver a hacerla (Fotografía 7). A veces, sin darnos cuenta, llegábamos hasta el final y cuando no funcionaba, no siempre nos quedaba claro dónde estaba el error, entonces la tarea era volver sobre cada puntada hasta encontrar el problema. Algunas bordadoras o tejedoras expertas logran identificar los errores a la vista, pero en el hacer textil que se aprende, como es el caso del que está a la base de estas exploraciones textiles-digitales, la experticia se gana desenvolviendo el movimiento.

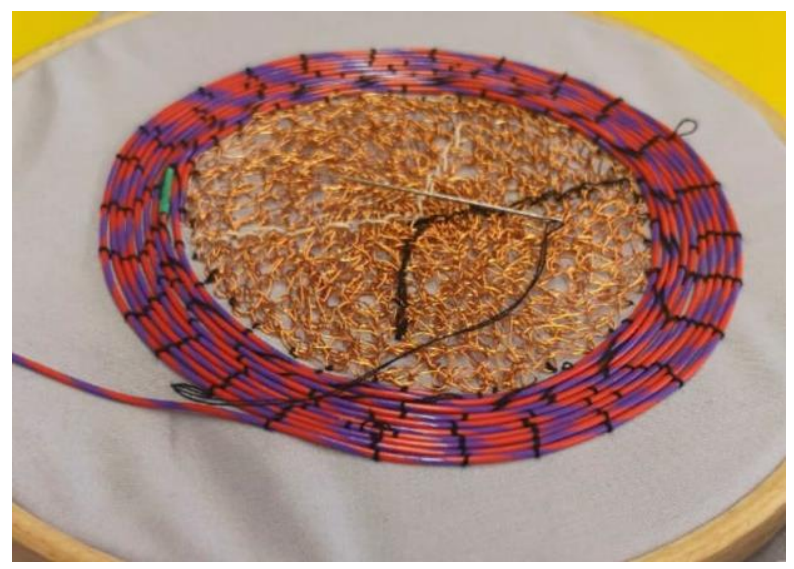

Fotografía 7. Parlante artesanal en proceso de elaboración. En la fotografía se pueden observar algunos remiendos, como la unión verde cuando se acabó el cable o como el cambio en el estilo de la elaboración de la espiral (tejido en el centro, bordado en la periferia). Fuente: Registro visual de nuestro trabajo de campo. 
En nuestro aprender lo textil, deshacer era una constante. Intentábamos entender cómo se hacía una puntada para poder comprender la forma en que ella podría juntarse a otra y así imaginar posibles nuevas combinaciones de las mismas que luego pudieran representarse computacionalmente. Para esto una maestra bordadora nos indicaba como hacer el movimiento y nos invitaba a repetirlo, "hágale que si nos sale mal desbaratamos y lo volvemos a hacer" decía, sabiendo, por un lado, que esa era la forma en que ella también procedía y, por el otro, como una manera de convocar la paciencia, para no desistir frente a las frustraciones que trae ese aprender cuidadoso y repetitivo. En ese contexto el tiempo transcurre. El hacer textil que acompaña los tiempos cotidianos de quienes lo realizan tiene la potencia de desacelerar los ritmos corporales, generando espacios de silencio que son propios, pero que activan la escucha de lo que nos pasa adentro (lo que sentimos, lo que pensamos), como de lo que ocurre allí en el devenir inmediato de los movimientos vinculantes ${ }^{8}$.

El tiempo es un factor determinante en los procesos de ingeniería. La revolución industrial, cuyo inicio estuvo estrechamente ligado con el sector textil, estuvo determinada por la necesidad de hacer procesos que produjeran más resultados en menos tiempo y haciendo uso de menos recursos, más eficientes (Styles, 2016). Es así que en los escenarios de ingeniería se busca continuamente la eficiencia, no solamente en los artefactos, sino en las personas que participan en esos escenarios. El diseño se convierte en una estrategia para anticipar los resultados, reducir errores, y evitar tiempo en hacer-deshacer-rehacer. A los estudiantes de ingeniería se les solicita definir unos objetivos claros antes de emprender un proyecto (anticipar), y luego se les evalúa con base en el cumplimiento o no de esos objetivos (Gonzalez Rivera et al., 2016).

${ }^{8}$ Es de señalar que esta escucha y vinculación no sólo genera paz, también puede dar cabida a la frustración y la rabia, que se acentúan justamente por el proceso de desaceleración. 
En este contexto, participar en los espacios de exploración lentos y repetitivos con maestras bordadoras controvertía la manera tradicional en que hacíamos ingeniería: no se trataba de obtener resultados eficientes sin cometer errores, sino de encontrar momentos para hacer juntas, sin consultar el reloj, con el único propósito de estar allí. Cuando ocurrían errores se convertían en una oportunidad para deshacer y aprender sobre lo deshecho. Los errores, aunque se suelen esconder o borrar en los resultados de la ingeniería, son en realidad parte de su cotidianidad. Así como una bordadora experta es capaz de detectar errores en lenguajes textiles, que no son detectables por alguien no experto; una persona que programa tiene la capacidad de leer lenguajes computacionales y encontrar errores lógicos escondidos (llamados bugs). De hecho se dice la principal tarea en la programación de computadoras es encontrar errores (Thompson, 2019). Así, programar computadores era una habilidad que inicialmente se asociaba con labores textiles como el tejido o la costura, porque requería ser meticulosa, hacer repeticiones, concentrarse, detectar errores, ir y volver. Es allí que cobra sentido encontrar que fueron las mujeres, tejedoras, costureras, las pioneras en el desarrollo de software. Reconocer las temporalidades de ese trabajo cuidadoso se revela en la exploración cotidiana de las materialidades textiles con ellas. En ese encuentro la ingeniería desacelera sus objetivos de eficiencia y se abre paso para reconocer su propia historia y allí pensarse distinta.

\section{Recogiendo puntadas}

En este artículo hemos buscado dar cuenta, desde dos movimientos, de las formas en que el hacer textil interfiere en la manera en que podemos diseñar tecnologías digitales. Iniciamos recogiendo ejemplos históricos y contemporáneos en los que estos oficios se entrelazan y confunden. Con ello quisimos volver a subrayar que las dicotomías entre estos haceres y saberes son ficciones sociales que están profundamente ancladas a dicotomías de género. La separación tajante entre lo textil y lo digital, que 
asocia a estas labores con lo femenino y lo masculino, respectivamente, desconociendo la dimensión artesanal y tecnológica de ambos, es una forma de olvidar que el primero es el origen del segundo, e invisibilizar estas genealogías es una manera más de continuar generizándolas. Nos ha interesado señalar, en esta primera parte, que las materializaciones textiles de artefactos que computan información son formas particulares de objetar estas jerarquías sociales que ordenan también nuestros cuerpos. Estas objeciones textiles a los órdenes de género no son de ahora, se anudan en los quipus incaicos, permiten diseñar textiles que encienden las máquinas de la revolución industrial, sostienen los primeros intentos de llegar a la luna y las búsquedas contemporáneas por que los procesadores puedan hacerse manualmente e incorporarse en telas, embelleciéndolas.

En lo contemporáneo, lo textil también objeta las formas de hacer digitales, por ejemplo, guiando el desarrollo de materialidades "inteligentes" como telas para interactuar directamente con información digital, sin requerir electrónica dura puesta sobre ellas. Proyectos como el de Elisabetta Masamuto (Roberts, 2019), en el que se investiga el tejido como una forma de programación y la lana como un material topológico programable en la creación de estructuras complejas, son ejemplo de estas objeciones contemporáneas. De acuerdo con la narración de Masamuto, su interés en la lana como material programable nace con un hacer textil que realizaba desde niña y que le permitió preguntarse y comprender desde la materialidad, que, por ejemplo, si bien una hebra de lana es rígida, cuando se teje con ciertos patrones y puntadas se pueden lograr diferentes propiedades de elasticidad. La lana es un material, mientras que el tejido de la lana es una materialidad que permite cuestionarse sobre el hacer computacional, la ingeniería de lo "inteligente" smart-, o el papel del cuerpo en el desarrollo de procesos y sistemas digitales. 
El recorrido y reflexión que realizamos en esta primera parte no ha pretendido ser exhaustivo ${ }^{9}$, sino servir de antesala a una reflexión etnográfica y transdisciplinar sobre la forma como estas objeciones textiles se hacen visibles en la cotidianidad del diseño de tecnologías digitales. En este segundo movimiento nos hemos adentrado en tres momentos de nuestro trabajo investigativo para reflexionar desde allí en la forma en que lo textil cuestiona el hacer del diseño en ingeniería: Le sostiene, le transforma y le ralentiza. Esta entrada desde los diálogos materiales con lo textil y lo digital nos ha permitido dar cuenta de los cuerpos que cuidan, de los lenguajes que conmueven y de los ritmos que acercan, procesos que no son ajenos a la experimentación ingenieril, pero que pueden llegar a serlo. En ese volver a mirar nuestros encuentros con lo material y las relaciones que de allí emergen, entre nosotras y entre lo digital y lo textil, se abren posibilidades investigativas y pedagógicas que pueden transformar la formación en ingeniería, no solo porque traen al presente referentes que contribuyen a reconocer que la ingeniería ha sido y puede ser un asunto de chicas, sino porque invita a un hacer ingeniería, en donde el hacer y el deshacer textil-digital, como metodologías inventivas para pensar lo computacional, puedan reconocerse como procesos pedagógicos conmovedores y encarnados, y por ello mismo importantes en la formación disciplinar.

Un componente importante de esta reflexión es la dimensión experimental que tienen los encuentros materiales a los que nos hemos referido. Las objeciones textiles de las que hablamos emergen de nuestro hacer y de nuestra reflexión sobre éste y en este sentido, no existen en sí, no preceden el encuentro material, como diría Barad, éste las produce. Esto es relevante pues nos llama a prestar atención a cómo se sostiene, transforma y ralentiza la producción generizada de la ingeniería como disciplina, a permanecer en esa reflexión, no suponiendo que estas son objeciones que trascienden los casos aquí presentados y

9 Para una mirada más exhaustiva y anglosajona de estas relaciones íntimas entre lo textil y lo digital ver Plant (1995); Rosner (2018). 
que pueden decirnos algo sobre el activismo feminista en torno a las tecnologías digitales de manera general. En este sentido este artículo vuelve a subrayar preguntas que los estudios feministas de la tecnología vienen haciendo desde sus inicios, y lo hace trayendo a colación respuestas situadas a las mismas. ¿Qué cuerpos (humanos y más que humanos) sostienen el trabajo de quienes diseñan ingeniería? ¿Cómo se relacionan estos cuerpos entre sí? ¿De qué manera podemos hacer visible esa labor de sostener el desarrollo de artefactos tecnológicos? ¿Cómo se han estandarizado estas relaciones corporales-materiales y qué consecuencias ha traído dicho proceso? ¿De qué manera al situar esa estandarización y comprender su dimensión encarnada puede conmoverse el hacer de la ingeniería? ¿Qué tiempos han sido producidos por esa estandarización? ¿Qué los activa? ¿Cuáles otros son posibles?

\section{Referencias bibliográficas}

ABET. Criteria for Accrediting Engineering Technology Programs, 2018 2019. IEEE Transactions on Software Engineering, SE-12, ABET, 2018, pp.733-743 [https://doi.org/10.1109/TSE.1986.6312975].

ANGUlo, Annuska; MARTíneZ, Miriam Mabel. El mensaje está en el Tejido. Ciudad de México, Futura Textos, 2016.

ARNOLD, Denise; ESPEJO, Elvira. El Textil Tridimensional: Pautas Hacia La Naturaleza Del Tejido Como Objeto y Como Sujeto. La Paz, Fundación Albó, Fundación Interamericana e Instituto de Lengua y Cultura Aymara, 2013.

BARAD, Karen. Posthumanist Performativity: Toward an Understanding of How Matter Comes to Matter. Signs: Journal of Women in Culture and Society 28(3), 2003, pp.801-831 [https://doi.org/10.1086/345321].

BASILI, Victor R; SELBY, Richard W.; HUtChENS, David H. Experimentation in Software Engineering. IEEE Transactions on Software Engineering, SE-12, 1986, pp.733-743 [https://doi.org/10.1109/TSE.1986.6312975]. 
CORTÉS-RICO, Laura. ApTUI Framework Para El Diseño Participativo de Interacciones Tangibles. Pontificia Universidad Javeriana, 2015 [http://pegasus.javeriana.edu.co/ P PI133-03-

ApTUI/docs/MemoriasApTUI_LauraCortés.pdf].

DedeOĞLU, Saniye. Visible Hands - Invisible Women: Garment Production in Turkey. Feminist Economics 16(4), 2010, pp.1-32 [https://doi.org/10.1080/13545701.2010.530606].

DIETER, George; SCHMIDT, Linda. Engineering Design. Edición 5. McGraw-Hill Education, 2012.

EBERT, Camila; HARLOW, Mary; ANDERSSON, Eva; BJERREGAARD, Lena. Traditional Textile Craft - an Intangible Cultural Heritage? Copenhagen, Centre for Textile Research, University of Copenhagen, 2014.

FernaEus, Ylva; JONSSON, Martin; THOLANDER, Jakob. Revisiting the Jacquard Loom: Threads of History and Current Patterns in HCI. Proceedings of the SIGCHI Conference on Human Factors in Computing Systems, 2012, [https://doi.org/10.1145/2207676.2208280].

pp.1593-1602

FILDES, Jonathan. Weaving the Way to the Moon. BBC, 2009 [http://news.bbc.co.uk/2/hi/technology/8148730.stm].

FOG, Lisbeth. Tecnología para bordar el conocimiento cartagüeño. El Espectador, January 1, 2016 [https://www.elespectador.com/noticias/actualidad/tecnologia-bordarel-conocimiento-cartagueeno-articulo-608512].

GallowAY, Anne. Emergent Media Technologies, Speculation, Expectation, and Human/Nonhuman Relations. Journal of Broadcasting \& Electronic Media, 57(1), 2013, pp.53-65 [https://doi.org/10.1080/08838151.2012.761705].

GonZÁlez-RiverA, Rafael; CortÉS-Rico, Laura; PÉREZ-Bustos, Tania; FRANCO-AvellanEDA, Manuel. Embroidering Engineering: A Case of Embodied Learning and Design of a Tangible User Interface. Engineering Studies 8(1), 2016, pp.48-65.

HARAWAY, Donna. Sowing Worlds: A Seed Bag for Terraforming with Earth Others. In: GREBOWICZ Margret; MERRICK, Helen (ed.). Beyond 
the Cyborg: Adventures with Haraway. New York, New York, USA: Columbia University Press, 2013, pp.137-146.

INGOLD, Tim. Lines: A Brief History. London, England, Routledge, 2007.

INTERNATIONAL LABOUR ORGANIZATION. Labour Practices in the Footwear, Leather, Textiles and Clothing Industries, Report for the Discussion at the Tripartite Meeting on Labour Practices in the Footwear, Leather, Textiles and Clothing Industries. Geneva, 2000.

JONES, Dorothy. Defining Self and Others through Textile and Text. Women's Writing 8(3), 2001, pp.375-390 [https://doi.org/10.1080/09699080100200143].

LINDSTRÖM, Kristina; STÅHL, Åsa. Patchworking Ways of Knowing and Making. In: JEFFERIES, Janis; CONROY, Diana Wood; ClARK, Hazel (ed.) The Handbook of Textile Culture., London-New York, Bloomsbury Academic, 2016, pp.63-78.

MÜLLER, Ruth; KENNEY, Martha. Agential Conversations: Interviewing Postdoctoral Life Scientist and the Politics of Mundane Research Practices. Science as Culture 34(4), 2014, pp.537-559 [https://doi.org/DOI: 10.10807/09505431.2014.916670].

NORDÅs, Hildegunn Kyvik. The Global Textile and Clothing Industry Post the Agreement on Textiles and Clothing. Geneva, WTO Publications, 2004.

PATT, Yale. Requirements, Bottlenecks, and Good Fortune: Agents for Microprocessor Evolution. IEEE, 89, 2001, pp.1553-1559 [https://doi.org/10.1109/5.964437].

PERNER-Wilson, Hannah. Fabric Speakers. How to Get What You Want. 2011 [https:/www.kobakant.at/DIY/?p=2936].

PÉREZ-Bustos, Tania. El Tejido Como Conocimiento, El Conocimiento Como Tejido: Reflexiones Feministas En Torno a La Agencia de Las Materialidades. Revista Colombiana de Sociología, July 2016 [https://doi.org/10.15446/rcs.v39n2.58970].

PÉREZ-Bustos, Tania. Hilvanar Tecnologías Digitales y Procesos de Tejido o Costura Artesanal: Una Revisión Crítica de Prácticas. Signo y Pensamiento 36(70), 2017 , [https://doi.org/10.11144//Javeriana.syp36-70.htdp].

pp.14 
PÉREZ-Bustos, Tania; CortÉS-Rico, Laura; MÁRQuez-GutiérReZ, Sara. Giving Life to a TUI through Inspiration from Colombian Calado Embroidery and Its Materialities. In 4S Anual Meeting, 1-12, Denver, 2015.

PÉrez-Bustos, Tania; MÁrquez, Sara. Destejiendo Puntos de Vista Feministas. Reflexiones Metodológicas Desde La Etnografía Del Diseño de Una Tecnología. Revista Iberoamericana de Ciencia Tecnología y Sociedad 10(31), 2016, pp.1-18.

PéreZ-Bustos, Tania; TOBAR-RoA, Victoria; MÁRquez-Gutierrez, Sara. Etnografías de Los Contactos. Reflexiones Feministas Sobre El Bordado Como Conocimiento. Antípoda. Revista de Antropología y Arqueología, n. 26, 2016, pp.47-66 [https://doi.org/http://dx.doi.org/10.7440/antipoda26.2016.02].

Plant, Sadie. The Future Looms: Weaving Women and Cybernetics. Body \& Society $1 \quad(3-4), \quad 1995, \quad$ pp.45-64 [https://doi.org/10.1177/1357034X95001003003].

Posch, Irene. The Embroidered Computer. Irene Posch, 2016 [http://www.ireneposch.net/the-embroidered-computer/].

PSARRA, Afroditi. Embroidered Bits, 2015 [http://afroditipsarra.com/index.php?/workshops/embroidered-bits/].

ROBERTS, Siobhan. Para esta física, la teoría de nudos requiere de mucho hilo. The New York Times, May 23, 2019 [https://www.nytimes.com/es/2019/05/23/tejer-teoria-de-nudosmatematicas/].

Romero-SÁnCHEZ, Amparo. La Utopía Postfeminista: Del ciberfeminismo al tecnofeminismo. Cuadernos Del Ateneo, n. 32, 2014, pp.156-169.

RoSNER, Daniela K. Critical Fabulations: Reworking the Methods and Margins of Design. Cambridge, MA, The MIT Press, 2018.

Rosner, Daniela K.; SHOREY, Samantha; CrAFT, Brock R.; REMICK, Helen. Making Core Memory: Design Inquiry into Gendered Legacies of Engineering and Craftwork. Proceedings of the $2018 \mathrm{CHI}$ Conference on Human Factors in Computing Systems, pp.531:1531:13. $\{\mathrm{CHI}\}$ '18. ACM, 2018 [https://doi.org/10.1145/3173574.3174105]. 
SCIENCE MUSEUM. Lovelace, Turing and the Invention of Computers. Objects and Stories. 2018 [https://www.sciencemuseum.org.uk/objects-and-stories/lovelaceturing-and-invention-computers].

STYLES, John. Fashion, Textiles and the Origins of Industrial Revolution. East Asian Journal of British History 5, 2016, pp.161-189 [https://www.academia.edu/20778558/Fashion_Textiles_and_the_Ori gins_of_Industrial_Revolution].

SuCHMAN, Lucy. Located Accountabilities in Technology Production. Scandinavian Journal of Information Systems 14(2), 2002, pp.91105.

SuCHMAN, Lucy. Agencies in Technology Design: Feminist Reconfigurations. 5th European Symposium on Gender \& ICT Digital Cultures: Participation - Empowerment - Diversity. University of Bremen, 2009.

ThIRUVATHUKAL, George. A Mini-History of Computing. Chicago, American Institute of Physics, College Park, 2011.

THOMPSON, Clive. The Secret History of Women in Coding. The New York Times, 2019

[https://www.nytimes.com/2019/02/13/magazine/women-codingcomputer-programming.html].

TIERRA LIBRE. QUIPU: Nudos Numéricos y Parlantes. Contadores En Acción. Quipucamayocs. 2008 [https://samanez9.wordpress.com/2008/10/01/contadores-en-accionquipucamayocs-quipu-nudos-numericos-y-parlantes/].

ToBAR-ROA, Victoria. Sobre Una Tela Deshilada. Colombia, 2015 [https://www.youtube.com/watch?v=4MZM4Bo5jRg].

TOMAYKO, James E. Computers Take Flight: A History of NASA's Pioneering Digital Fly-By-Wire Project. Washington, NASA, 2000.

TuRING, Dermot. The Story of Computing. London, Arcturus, 2018.

VIDYASAGAR, V.P. Encyclopaedia of Textiles: Textile Automation. Delhi, India, Mittal Publications, 2000. 